\title{
Large Eddy Simulation of a Steady Circular Jet Issuing Into Quiescent Fluid
}

\author{
$\underline{\text { J.W. Jewkes }}^{\mathrm{a}}$, A. King ${ }^{\mathrm{a}}$, Y.M. Chung ${ }^{\mathrm{b}}$ \\ ${ }^{a}$ Fluid Dynamics Research Group, School of Civil and Mechanical Engineering, Curtin University of \\ Technology, Level 4, Building 204, GPO Box U1987, Perth WA 6845, Australia \\ ${ }^{\mathrm{b}}$ Centre for Scientific Computing, University of Warwick, Coventry, CV4 7AL, UK \\ Email: J.Jewkes@Curtin.edu.au
}

\begin{abstract}
In this paper we present preliminary results from a high-resolution large eddy simulation (LES) study which will establish whether jet inlet velocity conditions are of significance in separation control applications. We include orifice geometry, in order to allow realistic turbulent structures to naturally develop. Ultimately, we will simulate various momentum thicknesses, $(D / \theta=50,80,120$ and 180) at $R e_{j}=10^{4}$ where $R e_{j}=U_{j} D / \nu$, where $U_{j}$ is the jet inflow velocity and $D$ is the jet diameter, however this paper presents initial high-resolution LES results for a steady jet, $R e_{j}=10^{4}, D / \theta=50$. The $\approx 1.2 \times 10^{7}$ cell model was computed using the iVEC EPIC supercomputing cluster, and our local Beowulf cluster at Curtin University, $(12 \times$ Intel $i 7920$ quad core CPUs (= 48 cores)). Our results show promising agreement with validation data, and indicate that our model has the potential to produce useful and accurate data regarding the evolution of a steady turbulent jet issuing into quiescent air.
\end{abstract}

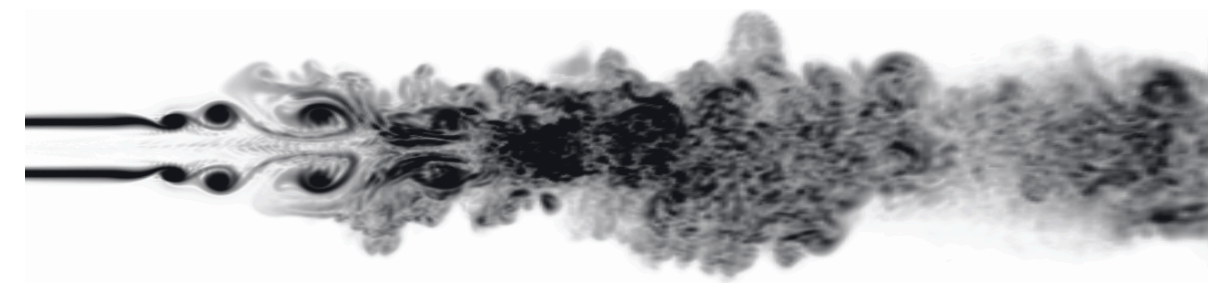

Centreline slice showing instantaneous vortical structures, vorticity magnitude scaled from 0 (white) to 10 (black).

Keywords: CFD, LES, Turbulent Flow, Jets, Flow Control 


\section{INTRODUCTION}

The circular jet is a 'kernel' fluid flow which can be seen utilised for propulsion, mixing, heating and cooling. Jets are common in the natural world, cephalopods such as squid and octopi propel themselves using water jets, and when threatened by predators, produce a jet of black ink (which mixes into an opaque cloud), to obfuscate their escape. Similarly, jets are utilised for the propulsion of high-speed boats, and microscopic jets are used in inkjet printers to form text and images. In this paper, our work is motivated by their potential application in boundary-layer flow separation control (Jewkes and Chung, 2010a,b, 2011). They can be used to produce streamwise 'corkscrew' vortices, which transport fluid from the high-momentum free-stream into the low-momentum near-wall region, and in this context they are usually described as 'vortex generating jets' (VGJs). For flow separation control, it is desirable to enhance momentum within the TBL, since an energised boundary layer is less prone to flow separation (Johnston, 1999). The favourable effect of these jets on separation is relatively well understood (Laval et al., 2010), however the underlying flow-physics remain poorly described.

Previous work has paid limited attention to the velocity profile and turbulent statistics of the VGJ. Experimentalists have often supplied fluid for the jet without careful consideration of the length and orientation of the pipe supplying it. In turn, simulations are often configured with arbitrary jet velocity profiles and turbulent statistics. The recent paper by Kim and Choi (2009) studied the effects of jet inflow conditions on the subsequent evolution of a turbulent jet, and showed that the jet characteristics significantly depend on the jet momentum thickness $(\theta)$ and the jet Reynolds number $\left(R e_{j}\right)$. Both of these properties could vary tremendously in a VGJ of a fixed mean velocity. In their work, the jet issues from the top of a chimney or column, which projects into the domain. In boundary layer flow control, jets typically issue from an orifice, flush with the wall. In Kim and Choi (2009), velocity boundary conditions are applied directly at the top of the column, however in our flow-control application, we are interested in the effect of jet velocity profile development within the orifice itself.

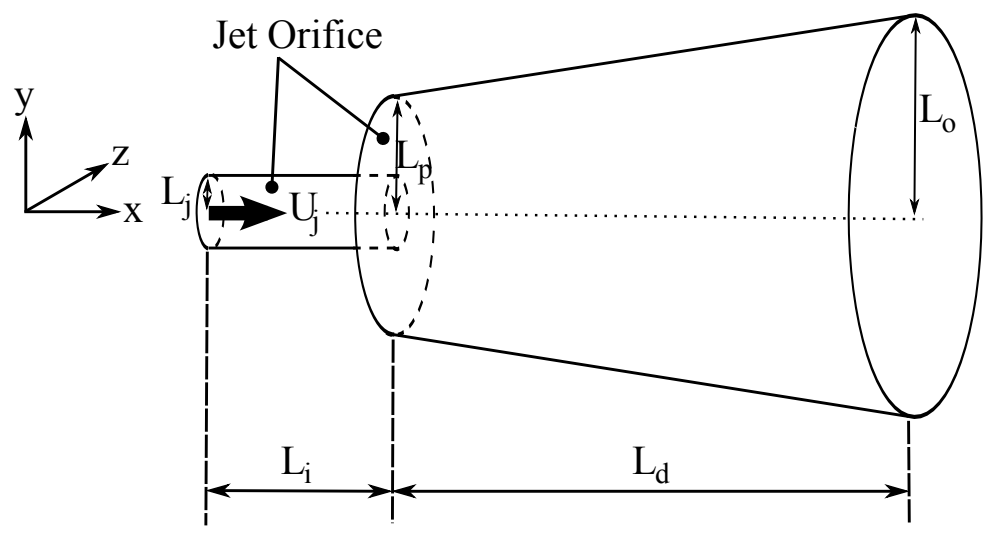

Figure 1. Schematic diagram showing the computational domain.

In this paper we present preliminary results from a wider study which will establish whether jet inlet velocity conditions are of significance in separation control applications. This simulation differs from Kim and Choi (2009) by modelling a jet issuing directly from the wall. We include orifice geometry, in order to allow realistic turbulent structures to develop naturally within the orifice (see Figure 1). Ultimately, we will simulate various momentum thicknesses, $\left(D / \theta=50,80,120\right.$ and 180) at $R e_{j}=10^{4}$ where $R e_{j}=U_{j} D / \nu$, where $U_{j}$ is the jet inflow velocity and $D$ is the jet diameter. This paper presents preliminary high-resolution LES results for a steady jet, $R e_{j}=10^{4}, D / \theta=50$. 


\section{Computational Details}

The filtered Navier-Stokes equations were solved using OpenFOAM ${ }^{\circledR}$, a library of C++ classes for CFD simulation. OpenFOAM ${ }^{\circledR}$ includes a well-tested and validated LES capability (Fureby et al., 1997a,b, 2000; Fureby, 2007; Baba-Ahmadi and Tabor, 2009; Bensow and Bark, 2010; Payri et al., 2010). A finite-volume method was used to discretise the filtered Navier-Stokes equations. The dependent variables were integrated over each cell, with Gauss' theorem applied to yield a set of discretised equations. Divergence terms were represented as fluxes across the cell faces, evaluated using centred second order interpolation and normalised variable diagram (NVD) derived interpolation (Jasak et al., 1999). The pressure implicit system of operators (PISO) algorithm (Issa, 1986) was applied, using the Rhie and Chow (1983) procedure. Time integration was carried out by the 2 nd order Crank-Nicholson scheme. The solution was performed implicitly by matrix inversion using an incomplete Cholesky conjugate gradient method. A custom-written dynamic subgrid-scale model was applied to calculate the Smagorinsky constant (Lilly, 1992). In this model, the Smagorinsky constant is dynamically computed based on the information provided by the resolved scales of motion. It is often used in boundary-layer simulation to improve the modelling of anisotropic turbulent structures that develop in near-wall regions (Jewkes et al., 2011).

Figure 1 shows an illustration (not to scale) of the computational domain used for this simulation. All length-scales are non-dimensionalised with respect to the $D$, the orifice diameter, velocities with respect to $U_{j}$, the velocity of the jet core region. The main simulation domain is $L_{d}=22.0$ in the streamwise (x) direction, the radius flared from $L_{p}=5.0$ to $L_{o}=7.0$ to allow for spanwise expansion of the jet plume. The inlet is $L_{i}=3.0 \mathrm{long}$, the orifice radius $L_{j}=0.5(=D / 2)$. The size and configuration of this domain is comparable to Kim and Choi (2009).

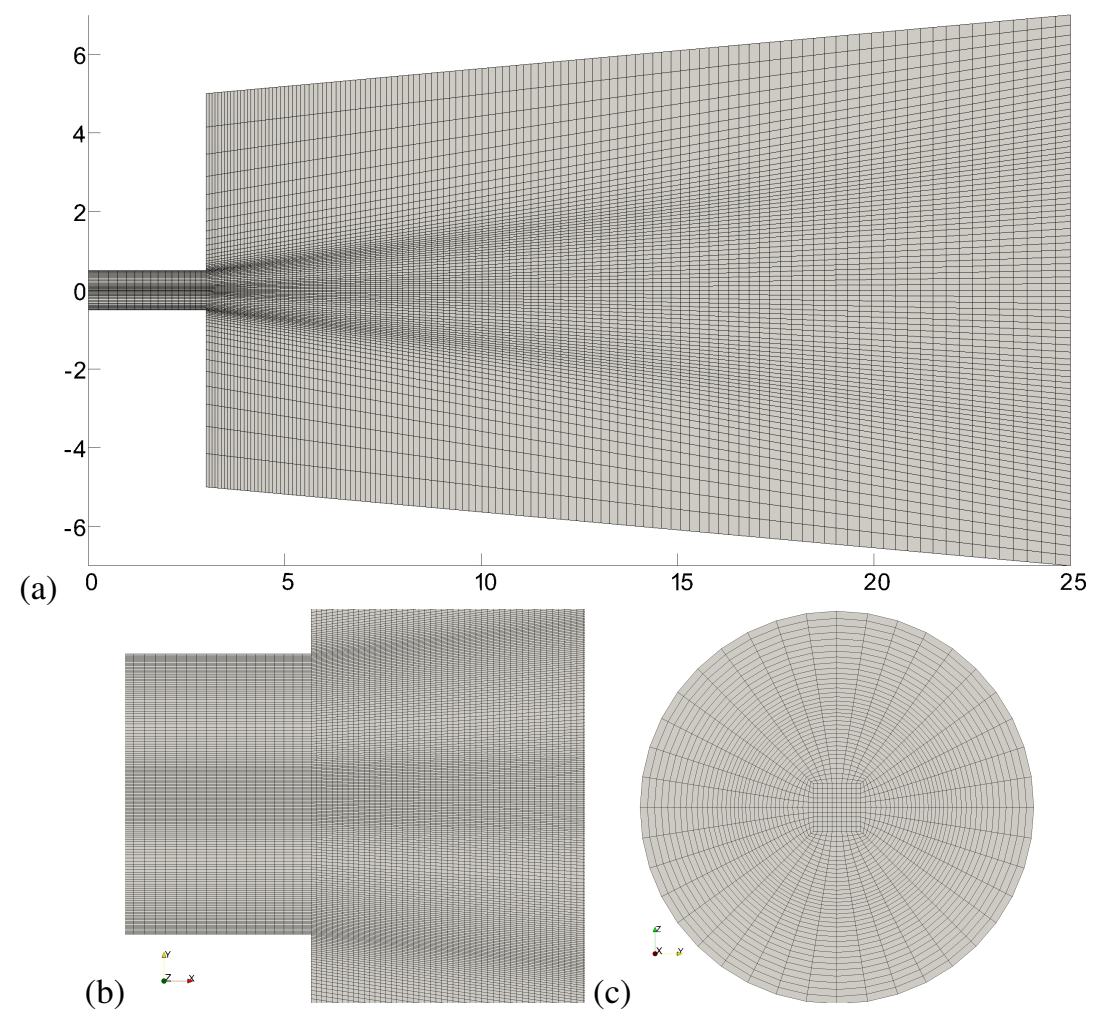

Figure 2. a) Centreline ( $x y)$ slice of the computational domain ( $1 / 64^{\text {th }}$ of full $3 D$ resolution); b) $x y$ close-up of the orifice (full resolution); c) $y z$ view of the domain at $x=25$ (1/64 ${ }^{\text {th }}$ of full $3 D$ resolution) 
The mesh consisted of $\approx 1.2 \times 10^{7}$ cells, comparable to the resolution used in Kim and Choi (2009); Figure 2 shows slices of this mesh. Figures $2 \mathrm{a} \& \mathrm{c}$ are shown at $1 / 64^{\text {th }}$ of the total $3 D$ resolution, and Figure $2 \mathrm{~b}$ is shown at full resolution. The mesh was created using blockMesh, a block-structured meshing utility in OpenFOAM ${ }^{\circledR}$. A 'butterfly' mesh configuration was used, and can most clearly be seen in Figure $2 \mathrm{c}$ (located at the end of the domain, $x / D=25.0$ ). Cells in the near-wall region of the jet are configured in uniform annular layers, graded such that maximum $y_{\text {wall }}^{+} \approx 1.0$, well within the viscous sublayer. The centre of the orifice resolves to a uniform hexahedral block. Figure $2 \mathrm{~b}$ shows a centreline slice of the mesh at full resolution, showing the heavy refinement in the orifice region. Figure $2 \mathrm{a}$ shows the expansion of mesh grading from the orifice to the far field.

A jet velocity boundary condition was applied at the inlet plane $(x=0)$, uniform in the jet core region. A $7^{\text {th }}$ power law boundary layer profile was applied around the edge of the circular inlet plane, in the nearwall region. Random fluctuations of $u_{j}=0.001 U_{j}$ were superimposed upon the profile at the beginning of each timestep. A 'fixedValue' (Dirichlet) boundary condition, $u_{i}=0$, was applied at the sides of the orifice, and at the wall (at $x=3$ ). A 'zeroGradient' (Neumann) boundary condition, $\partial u_{i} / \partial x=0$, was applied at the outflow boundaries.

The simulation was initialised with a quiescent flow field, and the simulation was run for $T=60 U_{j} / D$ (two flow-throughs) to allow transients to exit the domain. The Courant number was set such that it never exceeded 0.3. The iVEC EPIC supercomputing cluster was used to produce the initial $T=60$ solution, run-ons for time-averaging were produced using our own Beowulf cluster $(12 \times$ Intel $i 7920$ quad core CPUs $(=48$ cores $)$ ). Wall-time for $T=50 U_{j} / D$ of solution, running on our cluster was approximately 3 days.

\section{RESUlts}
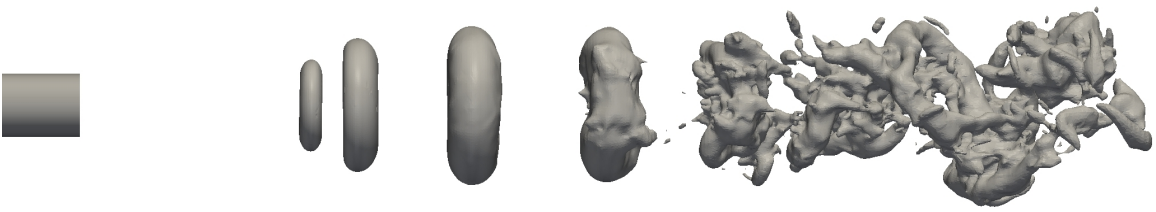

(a)

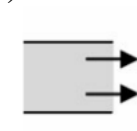

(b)

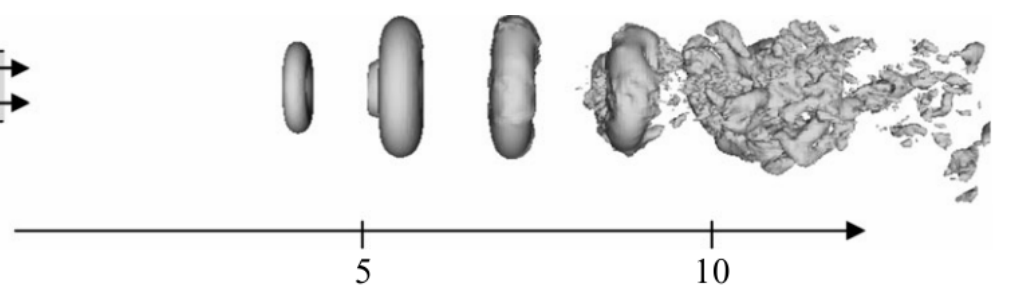

Figure 3. Instantaneous vortical structures at $R e_{j}=10^{4}, D / \theta=50$ : (a) Current Simulation; (b) Kim and Choi (2009). Iso-surfaces of $p / \rho U_{j}^{2}=-0.03$ are shown.

Figure 3 shows iso-surfaces of $p / \rho U_{j}^{2}=-0.03$, instantaneous vortical structures at $R e_{j}=10^{4}$, $D / \theta=50$ from Kim and Choi (2009), and the present study. Figure 3 demonstrates good qualitative agreement between the two simulations. As the jet column issues from the orifice, its shear-layer rapidly becoming unstable due to a Kelvin-Helmholtz instability. This instability causes the formation of coherent, axisymmetric vortex rings, which eventually begin to pair and break down into a fully saturated turbulent plume. This can perhaps be more clearly seen in Figure 4.

Figure 5 shows a graph showing mean axial velocity along the jet centreline, $R e_{j}=10^{4}, D / \theta=50$. The jet centreline velocity is considered one of the indices which represents the degree of jet mixing, through interaction between the shear layer, and the quiescent surrounding air. The velocity appears to decay in good agreement with Kim and Choi (2009), and Russ and Strykowski (1993). 


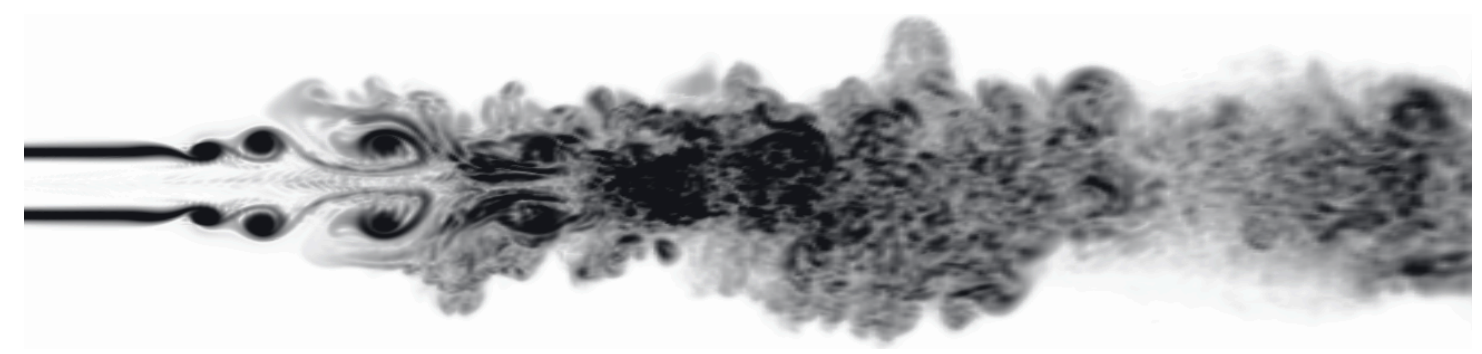

Figure 4. Centreline slice showing instantaneous vortical structures, vorticity magnitude scaled from 0 (white) to 10 (black)

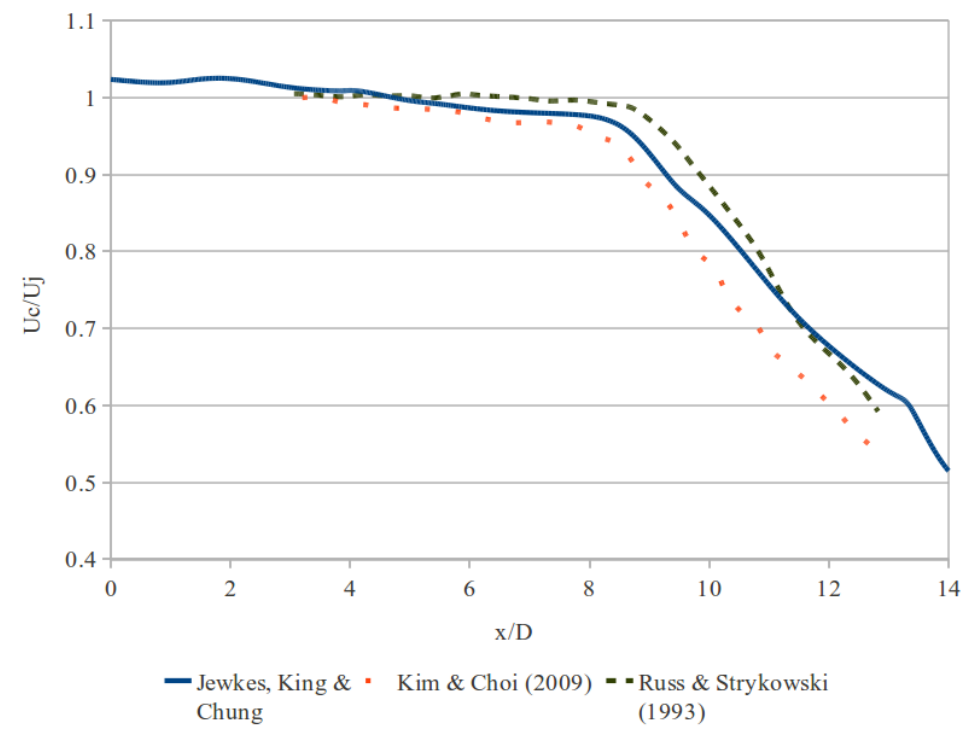

Figure 5. Mean axial velocity along the jet centreline, $R e_{j}=10^{4}, D / \theta=50$, including data from Kim and Choi (2009), and Russ and Strykowski (1993)

\section{Discussion}

These preliminary results indicate that our LES model has the potential to produce useful and accurate data, regarding the evolution of a steady turbulent jet issuing into quiescent air. However, there are some features of the model which we would like to improve, before we begin our full study. Figure 3 shows instantaneous vortical structures produced by the jet, coherent axisymmetric vortex rings. In Kim and Choi (2009), it is clear that these coherent vortical structures only occur in unperturbed jet simulations. Therefore we will provide improved turbulent statistics for the jet inlet, with the energy spectrum of the disturbance profiled such that it's frequency spectrum consists of the Kolmogorov spectrum in the inertial region, and the Pao spectrum in the dissipation region (Pope, 2003; Kim and Choi, 2009).

Figure $2 \mathrm{~b}$ shows the computational mesh in the region surrounding the jet orifice, and Figure $2 \mathrm{a}$ shows the expansion of mesh grading in the far field. The mesh grading between the orifice and the main simulation domain will be improved, and the expansion of mesh grading in the far field will be adjusted to bias cell refinement to within the jet shear layer. The full study will include grid convergence runs at double and half resolution, and the ratio of subgrid scale energy to resolved kinetic energy will be monitored to 
ensure the LES simulation is well resolved (Pope, 2003).

The overall aim of this study will be to determine the effect of the flow within the orifice, (velocity profile, turbulent statistics) upon the subsequent development of a turbulent jet issuing into quiescent air. This simulation differs from Kim and Choi (2009) by modelling a jet issuing directly from the wall. We include orifice geometry, in order to allow realistic turbulent structures to develop naturally within the orifice (see Figure 1). We will simulate various momentum thicknesses, $(D / \theta=50,80,120$ and 180) at $R e_{j}=10^{4}$ where $R e_{j}=U_{j} D / \nu$, where $U_{j}$ is the jet inflow velocity and $D$ is the jet diameter.

Ultimately we would like to perform a similar series of simulations in a VGJ in turbulent crossflow context (e.g., validated against the experimental work of Gopalan et al. (2004)), to establish the importance of the inlet boundary condition upon the subsequent development of a VGL in a boundary layer.

\section{REFERENCES}

Baba-Ahmadi, M. H. and G. R. Tabor (2009). Inlet conditions for LES using mapping and feedback control. Computers and Fluids 38(6), 1299-1311.

Bensow, R. and G. Bark (2010). Implicit les predictions of the cavitating flow on a propeller. ASME: Journal of Fluids Engineering 132, 0431302-1.

Fureby, C. (2007). Iles and les of complex engineering turbulent flows. ASME: Journal of Fluids Engineering $129,1514-23$.

Fureby, C., G. Tabor, H. Weller, and A. Gosman (1997a). A comparative study of sub grid scale models in homogeneous isotropic turbulence. Physics of Fluids 9(5), 1416-29.

Fureby, C., G. Tabor, H. Weller, and A. Gosman (1997b). Differential subgrid stress models in large eddy simulations. Physics of Fluids 9(11), 3578-80.

Fureby, C., G. Tabor, H. Weller, and A. Gosman (2000). Large eddy simulation of the flow around a square prism. AIAA Journal 38(3), 442-52.

Gopalan, S., B. M. Abraham, and J. Katz (2004). The structure of a jet in cross flow at low velocity ratios. Physics of Fluids 16, 2067-2087.

Issa, R. I. (1986). Solution of the implicitly discretised fluid flow equations by operator-splitting. Journal of Computational Physics 62, 40-65.

Jasak, H., H. Weller, and A. Gosman (1999). High resolution nvd differencing scheme for arbitrarily unstructured meshes. International Journal for Numerical Methods in Fluids 31, 431-39.

Jewkes, J. W. and Y. M. Chung (2010a). LES of a low velocity-ratio jet in a flat-plate boundary layer. In 6th Australasian Congress on Applied Mechanics, 12-15 December, Perth, Australia.

Jewkes, J. W. and Y. M. Chung (2010b). LES of a low velocity-ratio pitched and skewed jet in a flat-plate boundary layer. In 17th Australasian Fluid Mechanics Conference, 5-9 December, Auckland, New Zealand.

Jewkes, J. W. and Y. M. Chung (2011). Numerical investigation of the near-field flow structures produced by a vortex generating jet (under review). Physics of Fluids.

Jewkes, J. W., Y. M. Chung, and P. W. Carpenter (2011). Modification to a turbulent inflow generation method for boundary layer flows. AIAA Journal 49(1), 247-250.

Johnston, J. P. (1999). Pitched and skewed vortex generator jets for control of turbulent boundary layer separation: A review. In Proceedings of the 3rd ASME/JSME Joint Fluids Engineering Conference, 18-23 July, San Francisco, California.

Kim, J. and H. Choi (2009). Large eddy simulation of a circular jet: effect of inflow conditions on the near field. Journal of Fluid Mechanics 620, 383-411. 
J.W. Jewkes, A. King and Y.M. Chung; LES of a Steady Circular Jet.

Laval, J.-P., C. Braud, G. Fournier, and M. Stanislas (2010). Large-eddy simulations of control of a separated flow over a 2d bump by means of pulsed jets. Journal of Turbulence 11(52), 1-33.

Lilly, D. K. (1992). A proposed modification of the Germano subgrid-scale closure method. Physics of Fluids A 4(3), 633-635.

OpenFOAM ${ }^{\circledR}$ (2011). Version 2.0.0 users guide. OpenCFD ${ }^{\circledR}$ Ltd., http: / / www . openfoam. com/.

Payri, R., B. Tormos, J. Gimeno, and G. Bracho (2010). The potential of large eddy simulation (les) code for the modeling of flow in diesel injectors. Mathematical and Computer Modelling 52, 1151-60.

Pope, S. B. (2003). Turbulent Flows (First ed.). Cambridge University Press.

Rhie, C. and W. Chow (1983). A numerical study of the turbulent flow past an isolated airfoil with trailing edge separation. AIAA Journal 21(7), 1225-32.

Russ, S. and P. Strykowski (1993). Turbulent structure and entrainment in heated jets: the effect of initial conditions. Physics of Fluids A 5, 3216-25. 